\title{
Performance of a Practical Two-Step Detector for Non-Fluctuating Targets
}

\author{
Max Scharrenbroich*, Michael Zatman*, and Radu Balan ${ }^{\dagger}$ \\ *QinetiQ North America \\ Reston, Virginia \\ ${ }^{\dagger}$ Department of Mathematics \\ University of Maryland, College Park
}

\begin{abstract}
In this paper we analyze a two-step detection scheme for use in distributed sensor systems (e.g. statistical MIMO radar). The scheme arises when a data rate restriction forces each of the distributed systems to censor their detection statistics before sharing. We present the Neyman-Pearson (NP) two-step detection rule for a non-fluctuating target model (Swerling 0), which is non-linear and requires a priori knowledge of the target SNR. We then analyze the performance of a practical two-step detection rule under the non-fluctuating target model.
\end{abstract}

\section{INTRODUCTION}

Individual sensors are capable of producing detection statistics at a high data rate. When individual sensor platforms are aggregated to improve sensitivity, such as in statistical MIMO radar [1], [2], [3], the aggregate data rate can saturate both the communication and computational capacity of the system.

The two-step detection scheme arises when an aggregate data rate restriction is imposed on a distributed sensor system. One approach to restricting the aggregate data rate is to initially censor, or equivalently, threshold the detection statistics at each platform and share only those statistics that pass an initial threshold test (see Fig. 1). We refer to this initial threshold test as Stage 1 (first-stage detection) of the two-step detection scheme. After being shared, the detection statistics are passed to Stage 2 (second-stage detection) of the two-step detection scheme where they are integrated and compared to a final threshold corresponding to the desired overall probability of false alarm.

Under the assumption that the number of actual targets is small compared to the number of detection cells, most of the first-stage detection statistics are due to false alarms from noise. Thus an individual platform's data rate is directly proportional to the first-stage probability of false alarm (PFA). By reducing each platform's first-stage PFA the aggregate data rate is reduced.

To illustrate, if a single platform is producing detection statistics at a data rate of $100 \mathrm{Mb} / \mathrm{s}$, by initially thresholding the detection statistics such that the Stage 1 PFA is $10^{-3}$, the data rate into Stage 2 is reduced to approximately $100 \mathrm{~Kb} / \mathrm{s}$.

This work was sponsored by the Office of Naval Research under contract number N00178-04-D-4030-EH02. All views in this paper are those of the authors, and are not necessarily endorsed by the U.S. Navy.

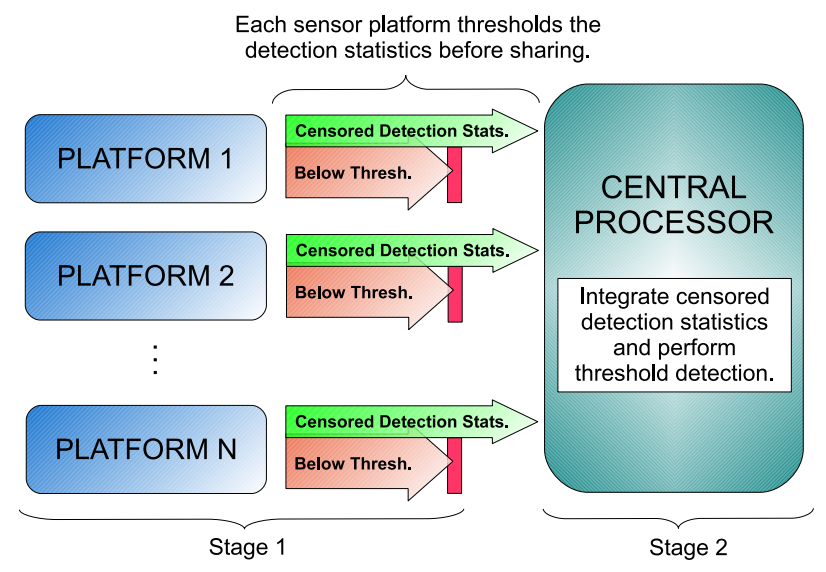

Fig. 1. Two-Step Detection Scheme

\section{Two-Step Detection Rule}

In [4] the Neyman-Pearson (NP) two-step detection rule (2SD) for the Swerling 2 (SW2) [5] fluctuating target model was derived and was shown to be clairvoyant [7] because of its dependence on a priori knowledge of the unknown target SNR. Despite being clairvoyant, [4] presented empirical evidence that the performance of the detector is only weakly dependent on this a priori knowledge. Thus, by assuming a reasonable target SNR, the detector becomes a practical one. Additionally, the detection rule is linear, making threshold selection and performance analysis straightforward.

In this section we complement the SW2 results in [4] by presenting the NP two-step detection rule for the Swerling 0 (SW0) [5] target model.

\section{A. General NP Two-Step Detection Rule}

In [4] the general NP two-step detection rule was derived based on the following assumptions:

i) Noise at each sensor is independent and identically distributed (IID).

ii) The target SNR measured by each sensor is also IID.

iii) The detection cells for each platform align exactly (i.e. there are no registration errors). 
The general NP two-step detection rule is to decide $H_{1}$ (the hypothesis that a target is present) if:

$$
\begin{aligned}
\sum_{x_{k}>T_{1}}\{ & \left.\ln \left(p\left(x_{k} \mid H_{1}\right)\right)-\ln \left(p\left(x_{k} \mid H_{0}\right)\right)\right\} \\
& +(N-L) \ln \left(\frac{1-P_{d 1}}{1-P_{f a 1}}\right) \stackrel{H_{1}}{>} \ln (\lambda),
\end{aligned}
$$

where $p\left(x_{k} \mid H_{i}\right)$ is the PDF of platform $k$ 's shared detection statistic, $x_{k}$, conditioned on either $i=0$ (noise only), or $i=1$ (target present), $N$ is the total number of participating platforms, $L$, the detection level, is the number of sensors passing along their first-stage detection statistics ( $L=\sum_{k=1}^{N} 1\left\{x_{k}>T_{1}\right\}$ ), $P_{f a 1}$ is the first-stage PFA, $P_{d 1}$ is the first-stage probability of detection (PD), $T_{1}$ is the first-stage threshold associated with $P_{f a 1}$ and $\lambda$ is a detection threshold.

\section{B. Swerling O NP Two-Step Detection Rule}

In the case of the SW0 target model, the return pulse has a Rician power distribution, and the non-coherent integration (using a square law detector) of $M$ such pulses yields a noncentral $\chi^{2}$ distribution:

$$
\begin{aligned}
p\left(x_{k} \mid H_{1}, \xi\right)= & \left(\frac{x_{k}}{M \xi}\right)^{\frac{(M-1)}{2}} e^{-\left(x_{k}+M \xi\right)} \\
& \times I_{M-1}\left(2 \sqrt{M \xi x_{k}}\right),
\end{aligned}
$$

where $\xi$ is the target SNR and $I_{\nu}(\cdot)$ is the modified Bessel function of the first kind.

As $\xi \rightarrow 0$, (2) becomes the noise-only case which is an Erlang density (or $\Gamma(M, 1)$ ):

$$
p\left(x_{k} \mid H_{0}\right)=\frac{x_{k}^{(M-1)} e^{-x_{k}}}{(M-1) !} .
$$

After inserting (2) and (3) into (1) we have the Swerling 0 NP two-step detection rule: decide that a target is present $\left(H_{1}\right)$ if

$$
\sum_{x_{k} \geq T_{1}} \alpha\left(x_{k}, \xi\right) \stackrel{H_{1}}{>} \eta+L \kappa(\xi) \equiv T_{2, L}(\xi)
$$

where $\eta$ is a threshold parameter (implicitly) dependent on $\xi$ and the desired overall (second-stage) probability of false alarm $\left(P_{f a 2}\right)$,

$$
\begin{array}{r}
\alpha\left(x_{k}, \xi\right) \equiv \ln \left(I_{M-1}\left(2 \sqrt{M \xi x_{k}}\right)\right) \\
-\frac{(M-1)}{2} \ln \left(x_{k}\right), \\
\kappa(\xi) \equiv \ln \left(\frac{1-P_{d 1}}{1-P_{f a 1}}\right)-\ln ((M-1) !) \\
+\frac{(M-1)}{2} \ln (M \xi)+M \xi,
\end{array}
$$

and $T_{2, L}(\xi)$ is the second-stage threshold for the $L^{t h}$ detection level. To be concise, the explicit dependence of $P_{d 1}$ on $\xi$ and $P_{f a 1}$ is omitted.

We now make several important observations: i) The NP detector is a clairvoyant detector since it is dependent on an unknown parameter $\xi$, the target SNR.

ii) The second-stage detection statistic is a non-linear combination the shared first-stage detection statistics.

iii) Assuming knowledge of $\xi$ there is only a single threshold parameter $\eta$ that needs to be found based on the desired $P_{f a 2}$.

\section{A Practical Two-Step Detection Rule}

Aside from being clairvoyant, the Swerling 0 NP two-step detector is also non-linear. This not only makes its real-world implementation impractical from a computational and system engineering stand-point, it also makes threshold selection and performance analysis difficult.

In the performance analysis that follows we will use the Swerling 2 NP two-step detection rule derived in [4]:

$$
\sum_{x_{k} \geq T_{1}} x_{k} \stackrel{H_{1}}{>} T_{2, L}(\xi),
$$

where $T_{2, L}$ is defined as in [4].

The major caveat of using the detection rule above is that observation (iii) above is no longer valid, making the process of optimal threshold selection non-obvious. In order to circumvent this issue we will select the second-stage thresholds using results from [4] by assuming the target is SW2.

\section{ANAlysis of PERFormance}

\section{A. Second-Stage Probability of False Alarm}

We refer the reader to [4] where we presented an expression for computing the overall probability of false-alarm $\left(P_{f a 2}\right)$ given a set of second-stage thresholds for the linear detector in (7).

\section{B. Second-Stage Probability of Detection}

For the linear detector in (7) the second-stage probability of detection $\left(P_{d 2}\right)$ takes the following form:

$$
P_{d 2}=\sum_{L=1}^{N}\left(\begin{array}{c}
N \\
L
\end{array}\right)\left(1-P_{d 1}\right)^{(N-L)}\left(P_{d 1}\right)^{L} F_{L}\left(T_{2, L}\right),
$$

where

$$
\begin{aligned}
F_{L}\left(T_{2, L}\right) \equiv & \operatorname{Pr}\left(\Sigma_{l=1}^{L} x_{(l)}>T_{2, L} \mid\right. \\
& \left.x_{(1)}>T_{1}, \ldots, x_{(L)}>T_{1}, H_{1}\right)
\end{aligned}
$$

is the complementary cumulative distribution function of the second-stage detection statistic given that $L$ of the first-stage detection statistics are greater than $T_{1}$ and a target is present $\left(H_{1}\right)$.

For the SW2 target model we were able to derive a closed form for (8) [4]. Because of the complexity of the lefttruncated distributions that (8) involves under the SW0 target model we resorted to an approximation using a shifted Gamma density and moment-matching:

$$
F_{L}\left(T_{2, L}\right) \approx \frac{1-\Gamma\left(T_{2, L} / \theta, k\right)}{\Gamma(k)}, \quad T_{2, L} \geq L T_{1},
$$


where $k \theta=\left(m_{1, L}-L T_{1}\right)$ and $k \theta^{2}=\left(m_{2, L}^{2}-m_{1, L}^{2}\right)^{1 / 2}$, with $m_{1, L} \equiv L m_{1}$ and $m_{2, L} \equiv L\left(m_{2}-(L-1) m_{1}^{2}\right)$, where $m_{1}$ and $m_{2}$ are the first and second moments of the left-truncated density shown in (2) and were computed using results from [8]. In our analyses the approximation proved comparable to Monte Carlo simulations of the true distribution.

\section{RESUlts}

In this section we present two parts each covering a different analysis of the practical 2SD (7) detection performance under the SW0 target model, where the second-stage detection thresholds were selected based on the results in [4] by assuming the target is SW2. In part A we present plots illustrating how $P_{d 2}$ varies with $P_{f a 1}$, and in part B we present a series of plots illustrating how $P_{d 2}$ varies with target SNR.

In our analysis we consider the Cooperative Networked Radar (CNR) [3] implementation of statistical MIMO radar. In this scenario each platform transmits a single pulse and noncoherently integrates its own pulse plus those transmitted by the other participating platforms prior to first-stage thresholding.

\section{A. $P_{d 2}$ vs. $P_{f a 1}$}

In the following we present two plots (Fig. 2 and 3). The first illustrates how $P_{d 2}$ varies with $P_{f a 1}$ using the practical detector (7) under the SW0 target model. The second plot shows the equivalent SNR loss due to first-stage thresholding (censoring). The SNR loss is defined as the additional SNR needed to achieve the single-stage detection performance using the two-step detector.

We analyze two CNR cases where the number of platforms, $N$, is 4 and 8 , respectively. In both cases each platform transmits a single pulse (thus the number of pulses integrated at each platform, $M$, is 4 and 8, respectively) and $P_{f a 2}=10^{-6}$. The target SNR for each case is selected based on a PD of 0.5 for the single-stage detector (the unrestricted case where $\left.P_{f a 1}=1\right)$ where the total number of pulses integrated is $M \times N=16$ and $M \times N=64$ for the $N=4$ and $N=8$ cases respectively, and the overall PFA is $10^{-6}$. In the $N=4$ and $N=8$ cases this translates into a target SNR of $2.3 \mathrm{~dB}$ and $-1.4 \mathrm{~dB}$ per pulse per platform respectively. Note that going from the $N=4$ to the $N=8$ case we are able to maintain a PD of 0.5 with a lower target SNR since we are integrating over more platforms.

Fig. 2 shows the performance of the practical 2SD versus $P_{f a 1}$ and also includes two curves as references for each CNR case: an upper curve for the unrestricted case where $P_{f a 1}=1$ (equivalent to a single-stage detector), labelled as $1 \mathrm{~S}$ and a lower curve for the single platform case with a PFA of $10^{-6}$ (best possible single platform performance given the same overall PFA), labelled as 1P.

In Fig. 2 we see that $P_{d 2}$ suffers steeper losses versus $P_{f a 1}$ with decreasing target SNR because with a lower SNR the first-stage censors actual targets more often. In the $N=4$ case a $P_{f a 1}=10^{-3}$ translates into a reduction of $P_{d 2}$ by about 0.3 which is equivalent to an SNR loss of approximately $1.1 \mathrm{~dB}$.

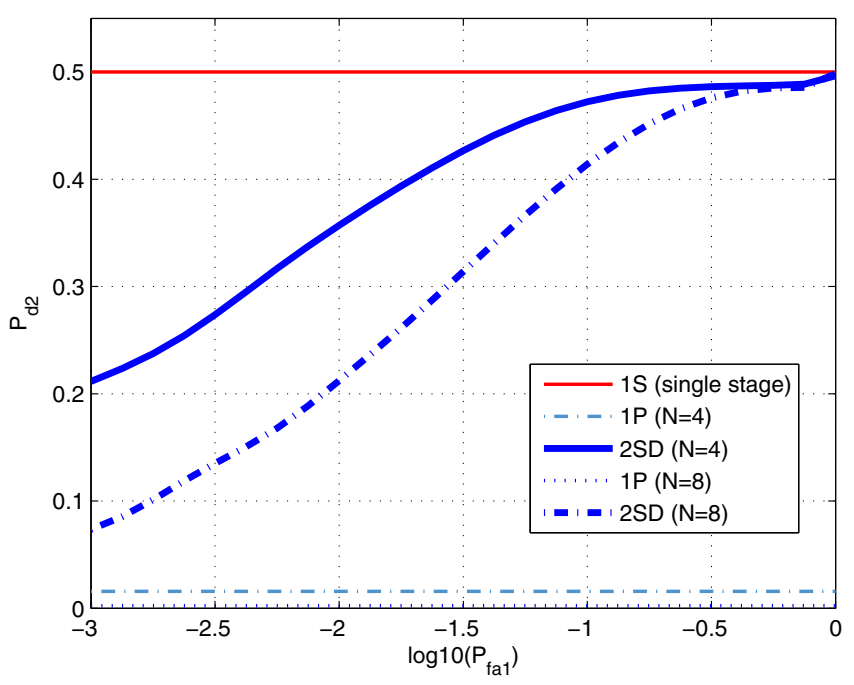

Fig. 2. 2SD detection performance curves illustrating how $P_{d 2}$ varies with $P_{f a 1}$ for $N=4$ and $N=8$ and a target SNR of $2.3 \mathrm{~dB}$ and $-1.4 \mathrm{~dB}$ per pulse per platform respectively.

In the $N=8$ case the same $P_{f a 1}$ reduces $P_{d 2}$ by slightly less than 0.4 which is equivalent to an SNR loss of approximately $1.8 \mathrm{~dB}$.

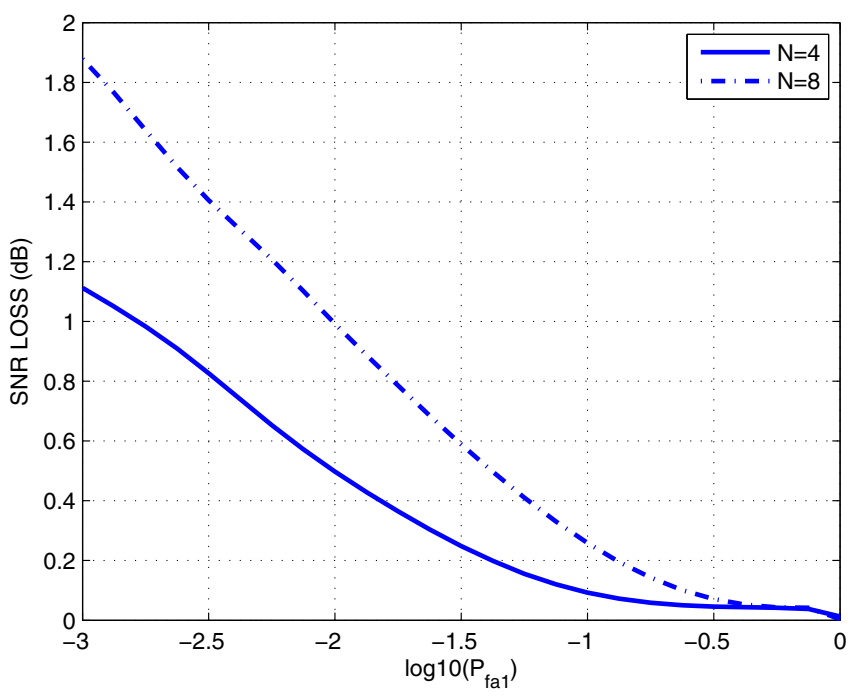

Fig. 3. Equivalent SNR loss as a function of $P_{f a 1}$.

\section{B. $P_{d 2}$ vs. Target $S N R$}

In this subsection we present two plots (Fig. 4 and 5) illustrating how $P_{d 2}$ varies with target SNR for the same two previous CNR cases $(N=4$ and 8$)$, with several values of $P_{f a 1} \in\left\{10^{-1}, 10^{-2}, 10^{-3}\right\}$ and $P_{f a 2}=10^{-6}$.

The focus of each plot is the comparison of a practical detector that can be easily implemented, to the optimal, yet unrealizable one, for the SW0 case. Hence, each plot displays two series: one series shows the performance of the practical 2SD (7) where the second-stage thresholds are fixed and were selected based on an assumed SNR yielding a PD of 0.5 
for the single-stage detector $(2.3 \mathrm{~dB}$ and $-1.4 \mathrm{~dB}$ per pulse per platform for $N=4$ and $N=8$ respectively). The second series shows the performance of the Swerling $0 \mathrm{NP}$ two-step detector (4) in which the second-stage thresholds were selected assuming prior knowledge of the target SNR. Because of the complexity of the Swerling 0 NP two-step detector, the second-stage thresholds and overall probability of detection were computed numerically using Monte-Carlo (MC) techniques and importance sampling.

For reference, these plots also include an upper curve for the unrestricted case and a lower curve for the single platform case.

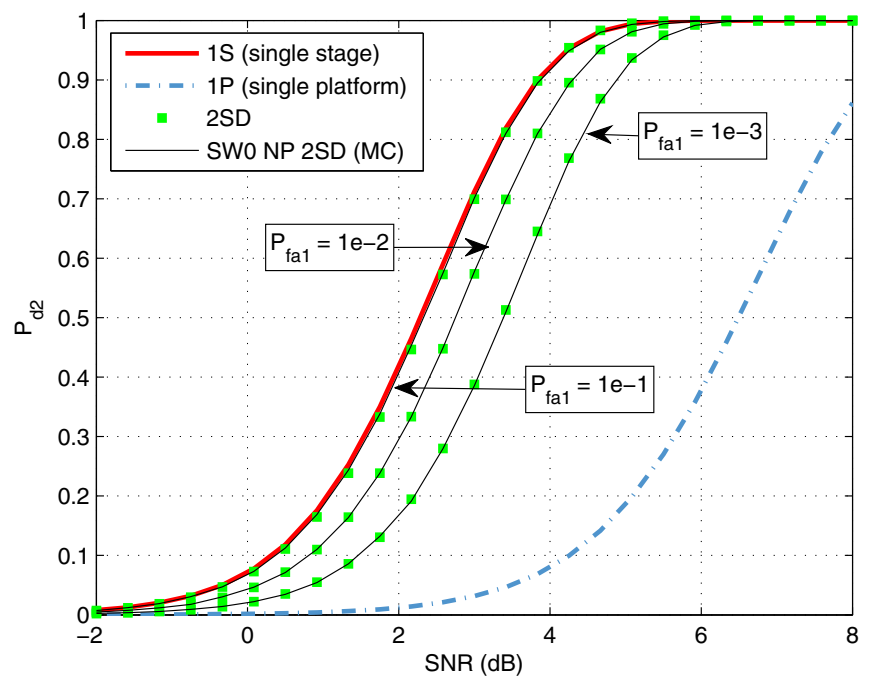

Fig. 4. Detection performance curves for a practical 2SD and the SW0 NP 2SD illustrating how $P_{d 2}$ varies with target SNR for the $N=4$ case.

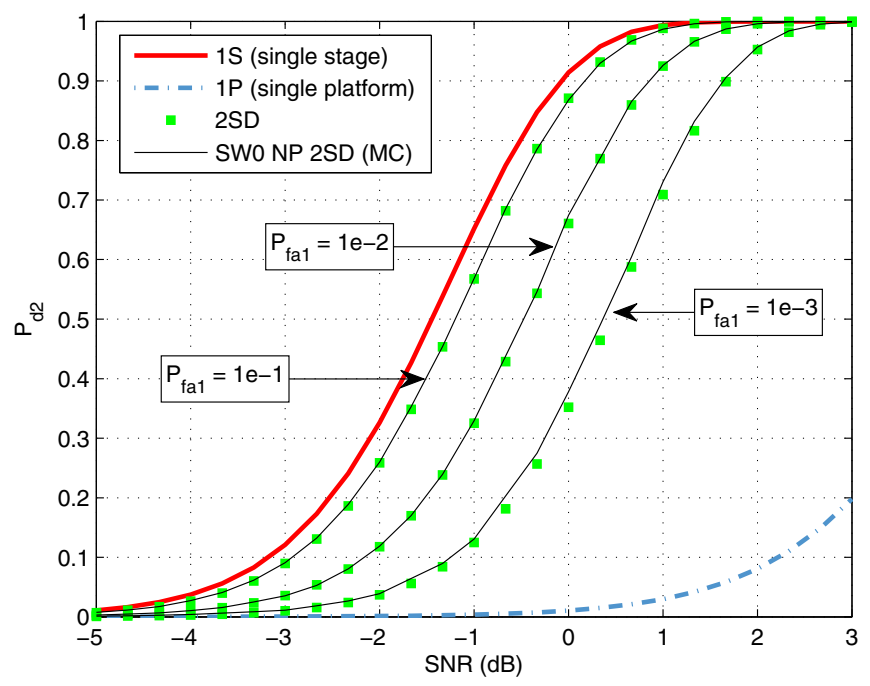

Fig. 5. Detection performance curves for a practical 2SD and the SW0 NP 2SD illustrating how $P_{d 2}$ varies with target SNR for the $N=8$ case.

It is interesting to note that in both the $N=4$ and $N=8$ cases the performance of the sub-optimal linear detector is close to that of the optimal one.

\section{CONCLUSION}

We have examined the two-step detection scheme that arises when practical data-rate limits are imposed on the Cooperative Networked Radar implementation of statistical MIMO radar. We have complimented our Swerling 2 NP two-step detection results presented in [4] and derived the Neyman-Pearson twostep detection rule in the case when the underlying target model is Swerling 0 (4), which is non-linear and requires a priori knowledge of the target SNR.

Due to the complexity of implementing the Swerling $0 \mathrm{NP}$ two-step detector our SW0 performance results are based on a practical linear detector (7), where the thresholds are selected by assuming the target is Swerling 2 [4] .

We have presented an approximation for the overall probability of detection for the Swerling 0 target model when using a linear two-step detection rule by using a shifted Gamma density and moment matching.

Our results echo those in [4] and indicate that the data rates in a distributed detection system can be reduced by several orders of magnitude using the two-step detection scheme without fully compromising the detection capability of the distributed sensor system.

Additionally, the results presented in Subsection B indicate that the performance of the linear detector is very close to optimal. Thus, we should expect the linear detector (with thresholds selected by assuming the target is SW2), to perform close to optimal over a range of fluctuating target models.

In future work we plan to compliment the SNR sensitivity results in [4] by investigating the loss in detection capability from using the linear detection scheme over the true optimal NP detection rule for the Swerling 0 target model.

\section{ACKNOWLEDGMENT}

The third author was partially supported by NSF DMS 0807896 and DMS 1109498.

\section{REFERENCES}

[1] J. Li and P. Stoica, Signal Processing for MIMO Radar, New Jersey: Wiley, 2008.

[2] Haimovich, A.M., Blum, R.S., and Cimini, L.J., "MIMO Radar with widely Separated Antennas", IEEE Signal Processing Magazine, Jan 2008.

[3] M.A. Zatman, "Cooperative Networked Radar," in Proc. Tri-Service Radar Symposium, July 2008.

[4] Scharrenbroich, M.F., Zatman, M.A., Balan, R.V., "Cooperative Radar Techniques: The Two-Step Detector", Asilomar Conf. Signals, Systems, Computers, Asilomar, 2011

[5] Swerling, P., "Probability of detection for fluctuating targets," Information Theory, IRE Transactions on, vol.6, no.2, pp.269-308, April 1960.

[6] Richards, M., Fundamentals of Radar Signal Processing, McGraw-Hill, New York 2005.

[7] S.M. Kay, Fundamentals of Statistical Signal Processing-II, Prentice-Hall, New Jersey 1998.

[8] Marchand, E., 1996, "Computing the Moments of a Truncated Noncentral Chi-Square Distribution", Journal of Statistical Computation and Simulation, 55: 1, 23-29. 